\title{
Cross-Country Path Finding using Hybrid approach of PSO and BBO
}

\author{
Harish Kundra*, Monica Sood" \\ *Assistant Professor, Rayat Institute of Engineering \& IT, Ralimajra, Punjab, INDIA, \\ ${ }^{\#}$ M.Tech Student, Rayat Institute of Engineering \& IT, Ralimajra, Punjab, INDIA
}

\begin{abstract}
In this paper we have proposed the implementation of optimized path. We are residing on a geographical area and there is no road. So path planning is a key factor to find out the optimized path to travel to destination. This paper describes a novel approach of autonomous navigation for outdoor vehicles which includes terrain mapping, obstacle detection and avoidance, and goal seeking in cross-country using Swarm Intelligence. This paper combines the strengths of both Particle Swarm optimization (PSO) for finding out the natural paths moreover keeping the obstacle detection from the satellite image, and Biogeography Based Optimization (BBO) algorithm for obstacle avoidance and move towards the shortest path to the goal. In this we have used the classified image. And find out the shortest path in order to find the cross country path planning phenomenon. We have assumed the source and destination in image and various paths which are called the natural paths generated by particle swarm optimization. The localization of islands positions has been done and through that the final optimized path which is called the shortest path has been find out for the destination. The HSI which is taken in islands is the shortest distance from the destination.
\end{abstract}

Keywords: Path planning, satellite image, terrain mapping, obstacle detection and avoidance, and Swarm Intelligence.

\section{INTRODUCTION}

Off-road autonomous navigation is one of the most challenging task from point of view of constraints on mobility, lack of environment structure, density of hazards and any prior information. Path planning is a task to generate a safest path connecting the start and the destination in a known or unknown environment in terms of the shortest path and obstacle avoidance. Our task is to navigate in cross-country without any prior information and to reach the goal through a safe, smooth and shortest path. In this paper, we are presenting the use of Particle Swarm Optimization (PSO) algorithm on satellite image for extraction of natural or human made objects called obstacles and Biogeography Based Optimization (BBO) algorithm for path planning.

In cross-country, in-accessible natural and human-made objects extracted from satellite image and smooth path planning can be implemented for various kinds of purposes, e.g. military, transportation, robot navigation, etc. Automatic obstacle detection from satellite image and path planning is an emerging problem and no existing software is able to perform the task reliably. In this paper, combined approach of PSO and BBO algorithms has been implemented on satellite image to achieve the goal.
PSO and BBO algorithms come under the broad category of Swarm Intelligence which has been emerged from the behavior of social insects. Social insects are usually characterized by their self organization and with the minimum communication or the absence of it. Every social insect individually is self-autonomous. They can obtain information about environment and interact with the remote insects or environment indirectly, by stigmergy. All these features characterize Swarm Intelligence. We can find these features in nature such as ant colonies, bird flocking, animal herding, fish schooling etc. The two most widely used Swarm Intelligence algorithms are Biogeography Based Optimization (BBO) and Particle Swarm Optimization (PSO).

The paper is organized into five sections. Following the introduction, a section illustrates brief review of Swarm Intelligence algorithms. The third section describes the methodology and pseudo-code of implementation. The fourth section reports the result of the case-study implemented. The last section summarizes the important findings.

\section{SATELLITE IMAGE, PARTICLE SWARM OPTIMIZATION, BIOGEOGRAPHY BASED OPTIMIZATION}

\section{A. Satellite Image}

Remote sensing is the science and art of obtaining information about an object, an area or phenomenon through the analyses of the data acquired by device that is not in contact with the object, area or phenomenon under investigation. Using various sensors, we remotely collect data that may be analyzed to obtain information about the objects, area, and phenomenon being investigated. The remotely collected data can be of many forms, including variations in force distribution or electromagnetic energy distributions, the two basic processes involved are DATA ACQUITIONS and DATA ANNALYSES.

The elements of data acquit ions are energy sources, propagation of energy through atmosphere, energy interactions with earth surface features, retransmission of energy through the atmosphere, airborne sensors, resulting in the generation of sensor data in pictorial of digital form. In short we use sensors to record variations in the way earth surface features and emit electromagnetic energy. The data analyses process involves examining the data using various viewing and interpretation devices to analyze pictorial data or a computer to analyze digital data. Reference data about the resources being studied are used when and where available to assist in the data analyses. With the aid of reference data, the analyst extracts information about the type, extent, location and condition of various resources over which the sensor data 
were collected. This information is then compiled in the form of hard copy maps, tables or as computer files that can be merged with other layers of information in geographical information system. Finally the information is presented to users who apply it to their decision making process.

Remote sensing affords us capability to literally see the invisible. It can be thought of as the eyes of many systems like GIS, providing repeated, synoptic visions of earth surfaces from an aerial or space vantage point. Satellite remote sensing images are representation of earth surfaces as seen from space. Remote sensing with multi-spectral satellite imagery is based on the concept that different features/objects constituting the land cover reflect the electromagnetic radiations over a wide range of wavelength in its own characteristic way. The satellite image of red band is shown in figure 1 which is an input image.

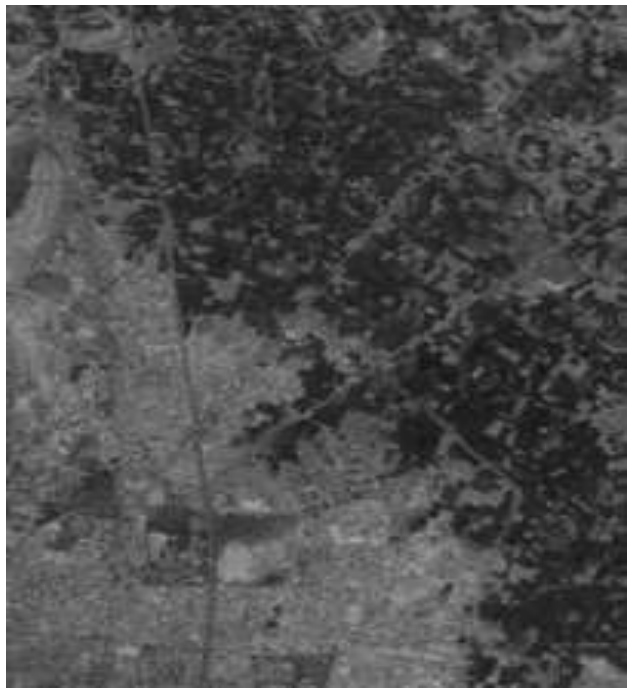

Fig 1. Red band satellite image (input image)

\section{B. Particle Swarm Optimization}

Particle swarm optimization is a stochastic, population-based computer algorithm for problem solving. It is a kind of swarm intelligence that is based on social-psychological principles and provides insights into social behavior, as well as contributing to engineering applications. The particle swarm optimization algorithm was first described in 1995 by James Kennedy and Russell C. Eberhart. The techniques have evolved greatly since then, and the original version of the algorithm is barely recognizable in current ones.

The particle swarm simulates social influence and social learning. A problem is given, and some way to evaluate a proposed solution to it exists in the form of a fitness function. The swarm is typically modeled by particles in multidimensional space that have a position and a velocity. These particles fly through hyperspace and have two essential reasoning capabilities: their memory of their own best position and knowledge of the global or their neighborhood's best. So a particle has the information to make a suitable change in its position and velocity.

\section{Biogeography Based Optimization}

The science of biogeography can be traced to the work of 19th century naturalists such as Alfred Wallace [1] and Charles Darwin [2]. Eugene Munroe was the first to develop mathematical models of biogeography in 1948 [3, 4], and Robert MacArthur and Edward Wilson were the first to extensively develop and publicize them in the 1960's .

Mathematical models of biogeography describe the migration, speciation, and extinction of species. Species migrate between islands. Note that the term "island" here is used descriptively rather than literally. That is, an island is any habitat that is geographically isolated from other habitats. In the classic sense of the term, an island is isolated from other habitats by water. But islands can also be habitats that are isolated by stretches of desert, rivers, impassable mountain ranges, predators, or other obstacles.

Islands that are well suited as residences for biological species are said to have a high island suitability index (ISI). Features that correlate with ISI include factors such as rainfall, diversity of vegetation, diversity of topographic features, land area, and temperature. The variables that characterize habitability are called suitability index variables (SIVs). SIVs can be considered the independent variables of the island, and ISI can be considered the variable.

Islands with a high ISI tend to have a large number of species, while those with a low ISI have a small number of species. Islands with a high ISI have many species that emigrate to nearby islands. The reason that emigration occurs from high ISI islands is due to the accumulation of random effects on a large population. For example, emigration could occur as animals ride flotsam to neighboring islands, or swim to neighboring islands. In the case of insects and birds, emigration can occur by flying or being carried by the wind.

Islands with a high ISI have a low immigration rate because they are already nearly saturated with species and cannot easily support new species. Islands with a low ISI have a high immigration rate because of their sparse populations, which allows a lot of room for additional species. The immigration of new species to low ISI islands might raise the ISI of the island because the suitability of a island is proportional to its biological diversity.

\section{Algorithms and Definitions}

Algorithm (1): Partial Immigration-Based BBO

The first approach is to base migration on immigration rates for each island, and probabilistically decide whether or not to immigrate each SIV (solution feature) independently.

$x_{i}(s)$ To denote the $s$ th feature of the $i$ th population member.

For each island $x_{i}$ 
For each SIVs

Use $\lambda_{\mathrm{I}}$ to probability decide whether to immigrate to $x_{i}(s)$

If immigrating then

Use $\mu$ to probabilistically select the emigrating island $x_{j}$

$x_{i}(\mathrm{~s}) \leftarrow x_{j}(\mathrm{~s})$

End if

next SIV

next island

Algorithm (2): Partial Emigration-Based BBO

The second approach is to base migration on emigration rates for each island, and probabilistically decide whether or not to immigrate each SIV independently. One generation of this approach can be described as follows:

For each island $x_{i}$

For each SIVs

Use $\mu_{i}$ to probability decide whether to emigrate to $x_{i}(s)$

If emigrating then

Use $\lambda$ to probabilistically select the immigrating island $x_{j}$

$x_{j}(s) \leftarrow x_{i}(s)$

End if

next SIV

next island

Algorithm (3): Single Immigration-Based BBO

The third approach is to base migration on immigration rates for each island, and probabilistically decide whether or not to immigrate a single randomly-chosen SIV.

For each island $x_{i}$

Use $\lambda_{i}$ to probabistically decide whether to immigrate to $x_{i}(s)$

If immigrating then

Pick a random SIVs
Use $\mu$ to probabilistically select the emigrating island $x_{j}$

$$
x_{i}(\mathrm{~s}) \leftarrow x_{j}(\mathrm{~s})
$$

End if

next island

Algorithm (4): Single Emigration-Based BBO

The fourth approach is to base migration on emigration rates for each island, and probabilistically decide whether or not to emigrate a single randomly-chosen SIV. One generation of this approach can be described as follows.

For each island $x_{i}$

Use $\mu_{i}$ to probabistically decide whether to emigrate to $x_{i}(s)$

If emigrating then

Pick a random SIVs

Use $\lambda$ to probabilistically select the immigrating island $x_{j}$

$x_{j}(\mathrm{~s}) \leftarrow x_{i}(\mathrm{~s})$

End if

next island

\section{METHODOLOGY}

Obstacle detection and path extraction of image procedure is to automatically categorize all pixels in image into roads, forest, barren land, water bodies and settlements. We extract the paths from image using PSO algorithm. To do this, appropriate threshold values have to be computed and then segmentation is performed. Now, obstacles have to be avoided and reach the goal through shortest path using BBO. In this phase, an algorithm for extraction of path and obstacle detection from satellite image is developed. This test image is presented various conditions under which the performance of our algorithm was evaluated.

\section{A. Detection of obstacles}

The algorithm is based on global threshold (average) using PSO. To obtain the desired results, the histograms of several of the scenes in the database containing Objects, were analyzed. From the analysis, we divided the histogram in five main regions. Region I start from the lowest intensity values to half of the midway of average intensity. For most of the cases, the intensity values on this region cover water bodies. Region II covers intensities that go from approximately half of the midway of the average intensity to midway of average intensity, and typically covers trees. Region III goes from midway of average intensity, to average intensity and covers bright gray objects such as highways. Region IV goes approximately from average intensity to midway of highest intensity and generally covers bright objects like 
buildings, road dividers, etc. Region $\mathrm{V}$ goes approximately from midway of highest intensity to highest intensity and generally covers barren land .

\section{B. Computing the Threshold using PSO}

To evaluate the threshold values, we created ten agents which scan their respective regions assigned to them from the image. Every agent scans the image row wise to find local best values for each row and eventually evaluates its global best for the assigned region. Among these regional global bests the agents select the most suitable candidate solution by communicating with each other. This is known as the Threshold value or the Global best.

\section{Path Planning Using BBO}

After the above operations, many possible paths are extracted amidst the cross-country. Now islands are created and randomly initialized from the starting point. They randomly chose different ways to reach the target. While moving to the target the species receive the obstacles and on way they detect and avoid the obstacles and reach to target finally. While moving towards the target they randomly select islands on each iteration on the basis of their HSI.The HSI is taken as shortest distance from the target. Obstacles coming in their way. On the whole, we have the safest and shortest path.

\section{Simulation}

The proposed algorithm is implemented with Matlab . The algorithm is based on combined approach of PSO and BBO applied on satellite image. Firstly red band satellite image was taken and calculated threshold using PSO for paths extraction and obstacle detection. To obtain the threshold value, agents were randomly initialized in a specified area and global best position was calculated. The paths extracted after applying PSO is shown in figure 2. Then obtained paths were refined using morphological operations to minimize the detection of shadows, trees and inconvenient areas. Figure 3 shows the refined image after morphological operations. Finally, BBO was implemented in which islands were initialized and they find the shortest path avoiding the obstacles from given source to destination.

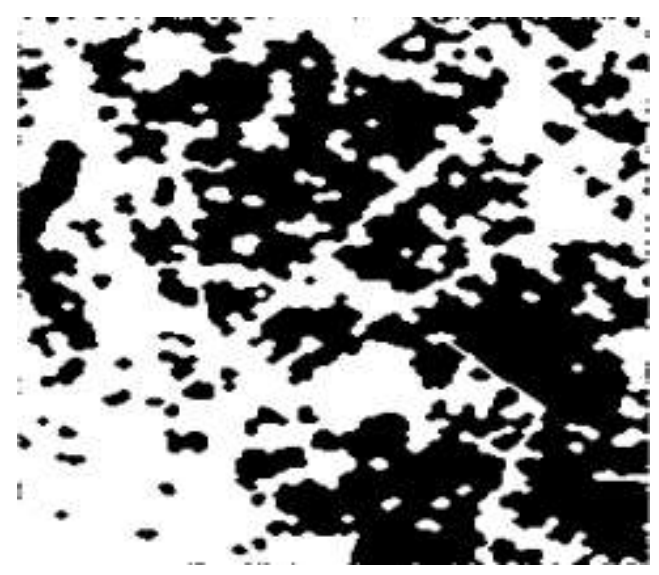

Fig2. Paths extracted after applying PSO

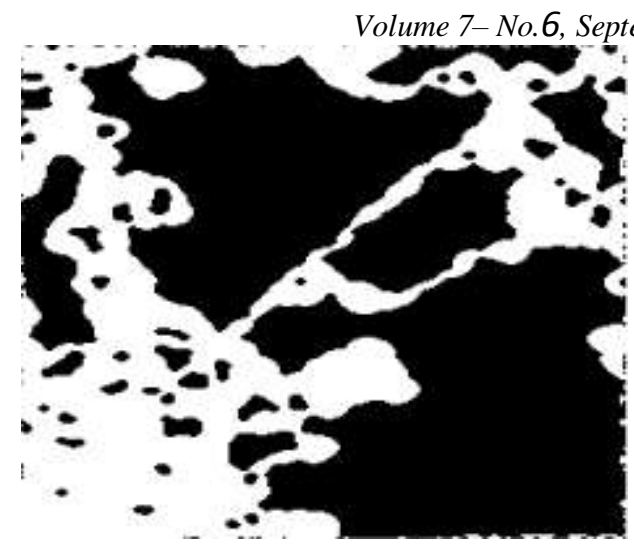

Fig 3. Refined paths after morphological operations

.The flowchart of the proposed algorithm is shown in figure 4. By following this algorithm we reached the target efficiently

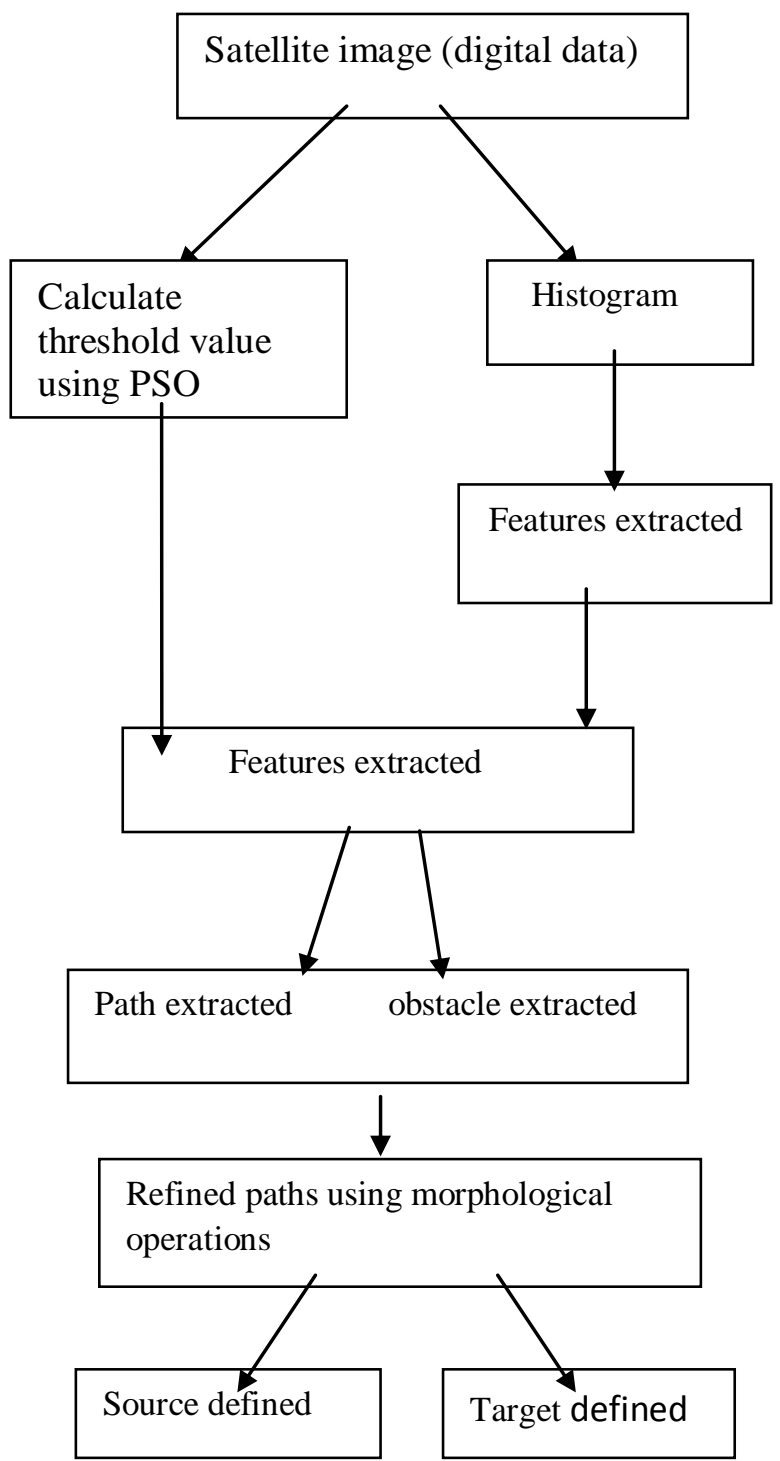




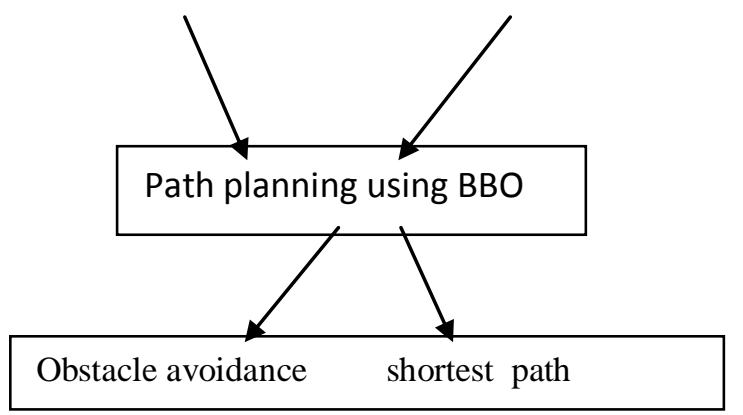

Fig4. Flowchart

The pseudo-code of the proposed algorithm is given

in figure 5:

Step 1: take satellite image as input image

Step 2: for each agent

Initialize the random position

Calculate the fitness value at that position

if fitness(n)<fitness(n-1)

then update best position

end-for

else not update

find global best position

for each agent end-for

update position and velocity

Step 3: calculate the threshold value (path extracted and obstacles detected)

Step 4: refine paths using morphological operations

Step 5: for island in biogeography

initialize the position

choose the next nearest path to the target

if path is obstacle

then choose the other nearest path(second

best path)

else move and update position of low HSI

Calculate

1) Emigration (if path is contained in previous path).

2) Immigration (if path is not contained in same previous path end-for

Step 6: find shortest path

\section{RESULTS}

Figure 6 shows the paths extracted and the shortest path from source to target from the satellite image using Swarm Intelligence.

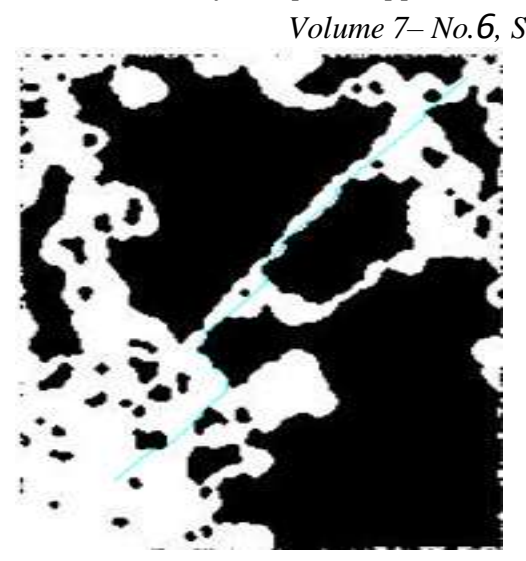

Fig 6. Final safest and shortest path to target

\section{CONCLUSION}

Enlightened by Swarm Intelligence, PSO path extraction and BBO path planning algorithm is proposed in this paper. Localization is used to achieve performance and experimental results suggest that in this case the role played by the heuristic information becomes less important. To make a path more smooth morphological operations were implemented to minimize the effects of shadows, trees and inconvenient areas. It can quickly plan an optimized path even in a complex environment. The results prove that proposed approach effectively extracts the obstacles and finds the shortest and safest path. The simulation results show that it's a simple, quick and efficient algorithm. This proposed algorithm may further be used for enemy detection, autonomous navigation, GIS mapping etc.

\section{REFERENCES}

1. Campbell, J. B. (2002), "Introduction to Remote Sensing”, Third edition, Taylor \& Francis, London, pp. 3,552 .

2. Kennedy,J.andEverhart,R.C.(1995), "Particle Swarm Optimization", In Proceedings of the IEEE International Conference on Neural Networks, 1995, Perth, Australia, vol. 4, pp. 1942-1948.

3. Macarthur, R. and Wilson, E. (1967), "The Theory of Biogeography", First Edition, Princeton University Press, New Jersey, pp.19-67.

4. Simon,D.(2008), “Biogeography-based optimization”, IEEE Transactions on Evolutionary Computation, vol. 12, no. 6, pp. 702-713. 